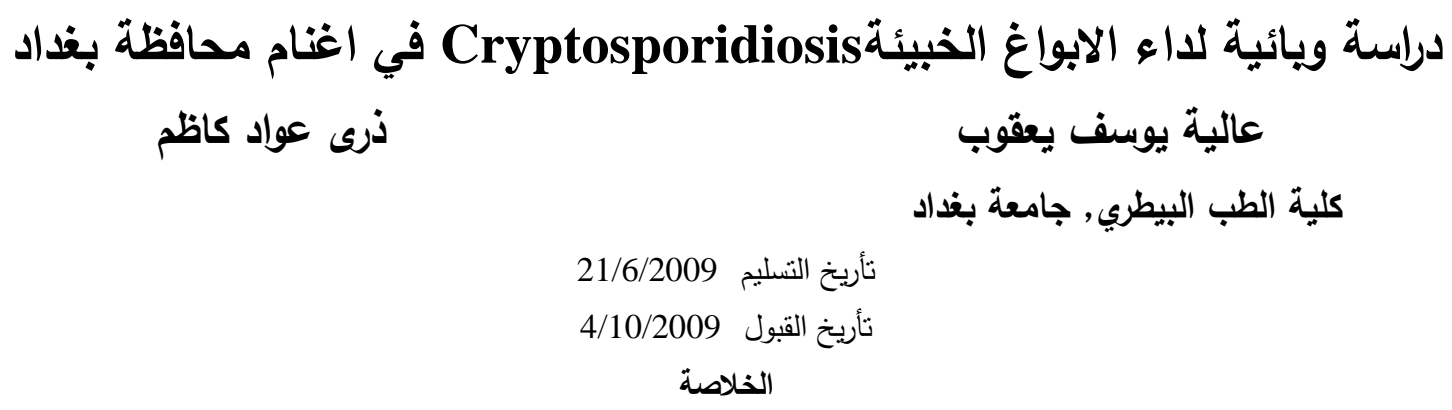

\title{
Epidemiological study of Cryptosporidiosis in sheep of Baghdad province
}

\section{Alia Yousif Yakoob College of Veterinary Medicine-Baghdad Uni. \\ Thura Awad Kathim}

\section{Summary}

To investigate the prevalence of cryptosporidiosis among neonatal lambs and sheep in different areas of Baghdad include (Alshaab, Alshoala ,Albayaa, Alalawy, Madenat alsader, Aldorah, Alhosaenea, Algazalea, Alrashdea) ,also the study investigated effect of sex ,age, season on the infected rate from December 2007 till August 2008.

The laboratory methods were used to identified the oocysts :Modifide Ziehl Neelsen stain and flotation with Shether's solution.

A total of 500 fecal samples were collected from animals at one day -two years old lambs and sheep, males and females

All fecal samples obtained from three groups of animals state: diarrhea , pasty and normal. 
The study recorded total rate of cryptosporidiosis infection $15.8 \%$ in neonatal lambs and sheep., the cryptosporidium oocysts isolated from animals appeared with Ziehl Neelsen stain as spherical oval shape, red in color containing the sporozoit and measured $4.3 \times 4.8 \mathrm{Um}$.

Infected rate differ according to the areas, the higher rate of infection $34.88 \%$ was recorded in Alshaab , while the lower rate $3.12 \%$ was recorded in Algazalea.

The result revealed higher rate of infection $34.95 \%$ was recorded in lambs less than month, while the lower rate $2.77 \%$ was recorded in animals at 6 to 7 month old.

No significant difference was noticed between two sex , the infection rate was $12.9 \%$ in males and $16.75 \%$ in females.

The result revealed there's no effect of fecal type on infection rate this study was recorded $18.61 \%$ in diarrheic animals, $14.68 \%$ in normal and $11.9 \%$ in animals with pasty feces.

In regard to seasonal variation, the higher rate of infection $34.88 \%$ was recorded in April, while the lower rate $3.12 \%$ in July.

المقدمة

داء الابواغ الخبيئة Cryptosporidiosis من الامراض الطفيلية التي تسبب التهاب المعدة والامعاء (1). والذي يسبيه احد

الاوالي الاكرية التابعة لجنس Cryptosporidium ،وهو من الامراض المشتركة اذ يصيب الانسان والعجول واللبائن الاخرى في جميع

(2) انحاء العالم

ينتشر المرض عن طريق المـاء والغذاء الملوثين بفضـلات الحيوانات المصـابة(4,3)، و تتقله الحشرات والقوارض المنزليـة الى

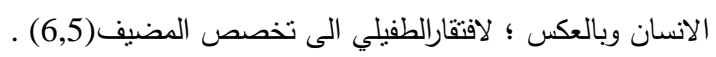

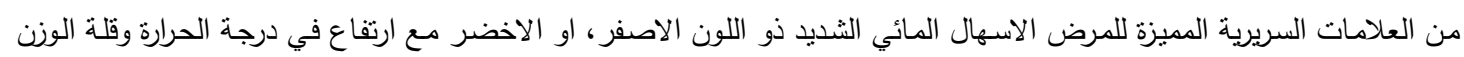

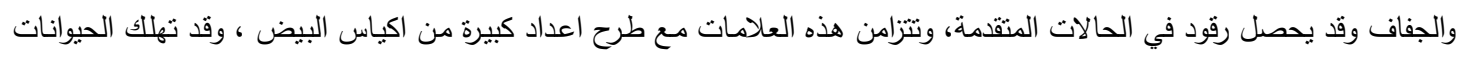

الصغيرة في حال ترافقت الاصابة مع قلة التغذية وسوء الادارة لاسيما في المواليد التي لا تعتمد على الرضاعة اعلى الطبيعية(7).

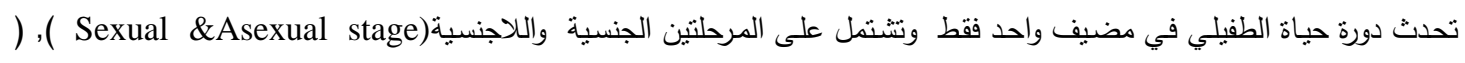

8)مدة حضانة المرض من( 7 -10)أيام، وتنتمر الحيوانات المصابة بطرح اكياس البيض اكثر من شهر (9).

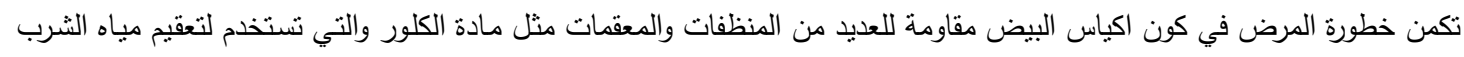

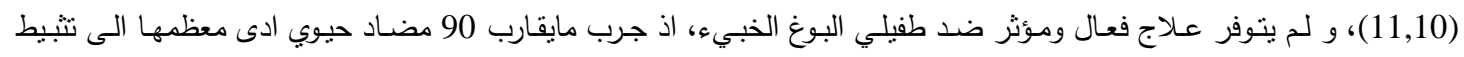

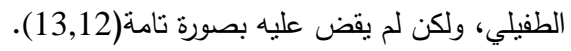

المواد وطرائق العمل

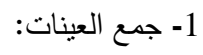

جمعت 500 عينة براز من حملان وأغنام بأعمار مختلفة نراوحت من ( يوم-سنتنين) ولكلا الجنسين للفترة من بداية شهر كانون

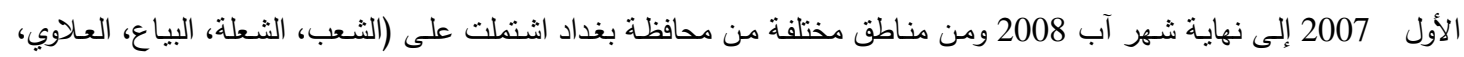

مدينة الصدر ، الدورة، الحسينية، الغزالية و الراثدية) , قسمت الحيوانات إلى ثلاث التهن مجموعات: الأولى, كانت تعاني من إسهال مائي

ذي لون اصفر أو اخضر مع هزال وضعف عام, الثانية: كان برازها عجينيا مع ضعف عله عام, الثالثة: كان برازها طبيعيا وبدون ظهور أي علامات سريريه.

2- الفحوصات المختبرية

الفحص العياني : تم فحص نماذج البراز عيانيا وسجل لون وقوام ورائحة البراز •

الفحص المجهري قسمت كل عينة إلى ثلاثة أجزاء:

الأول : حضرت منه مسحات خفيفة لغرض صبغها بصبغة الزيل نلسن المحورة Modified Zehil Neelsen Stain. الثاني: استخدم لإجراء فحص النطويف بالمحلول السكري المشبع Shether's Solution, الثالث: استخدم لعمل مسحات مباشرة

وصبغها بصبغة الايودين Lugol's Iodine

أولا : تحضير مسحات البراز وصبغها بصبغة الزيل نلسن المحورة

مزجت كمية قلبلة من البراز بقدر رأس عود الثقاب على شريحة زجاجية نظيفة مع قطرة من الماء ثم فرشت على المئ المساحة

الكلية للشريحة، وتركت لتجف بالهواء لمدة 10 دقائق مع مراعاة ترقيم الثريحة برقم العينة نفسها.

ثبتت المسحة بكحول الميثانول المركز (96\%) لمدة 5 دقائق وتركت لتجف. 
• أضيف إلى المسحة المنبتة صبغة الكاربول فيوكسن الحمراء المركزة وتركت لمدة 2-3 دقائق.

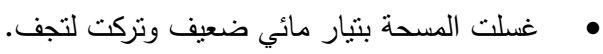

• قصرت المسحة بالكحول المحمض لمدة 30 ثانية، وغسلت بالماء ثم نركت بعدها لتجف.

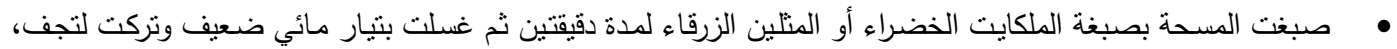

حيث حضرت من كل عينة مسحتين صبغت أحداهما بالمنلين الأزرق والأخرى بالملكايت.

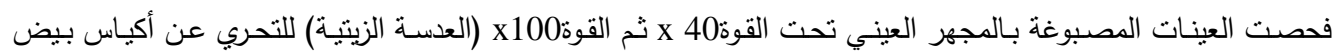

الطفيلي (14) .

ثانياً : التطويف بمحلول شيذر السكري وضـع 5 غرام من البراز في دورق زجاجي نظيف وأضيف له 10 مللتر من الماء المقطر ومزج بشكل جيد مع الرج ثم رشح

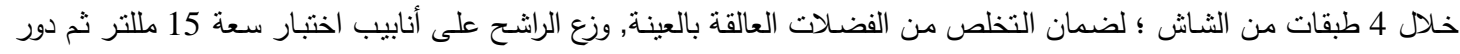

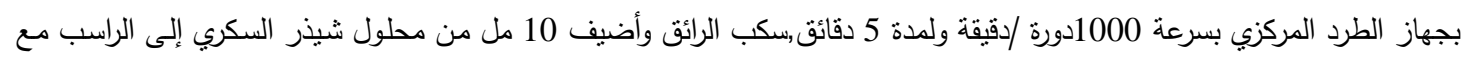

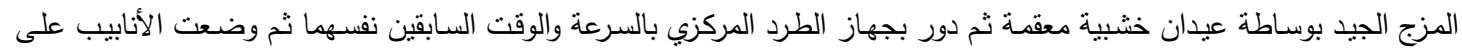

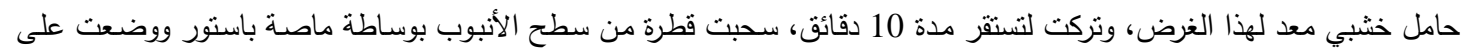
شريحة زجاجية وغطيت بغطاء الثريحة ثم فحصت بالمجر العيني تحت القونين(x 100, x 40 ) وحسب طريقة (15) .

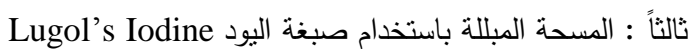
وضعت كمية قليلة من البراز على شريحة زجاجية واضيفت لها قطرة من محلول اليود ومزجت جيدا ثم غطيت بغطاء الثريحة،

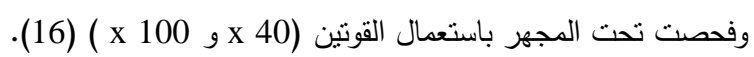
النتائج

جدول ( 4 ) نسبة الإصابة الكلية بطفيلي Cryptosporidium في الأغنام حيث بلغت 15.8\%

\begin{tabular}{|c|c|c|}
\hline النسبة المئوية\% & عدد الحيوانات المصابة & عدد الحيوانات المفحوصة \\
\hline 15.8 & 79 & 500 \\
\hline
\end{tabular}

جدول(5 ) أعداد ونسب الإصابة بطفيلي Cryptosporidium وحسب المنطقة حيث نلاحظ ارتفاع نسبة الاصابة في منطقة الثُبب وبلغت34.88\% فيما سجلت منطقة الغزالية ادنى نسبة اصابة وبلغت 3.12\%

\begin{tabular}{|c|c|c|c|}
\hline النسبة المئوية\%\% & عدد الحيوانات المصابة & عدد الحيوانات المفحوصة & المنطقة \\
\hline 34.88 & 15 & 43 & الشعب \\
\hline 11.66 & 7 & 60 & الشعلة \\
\hline 7.14 & 4 & 56 & البياع \\
\hline 12.5 & 3 & 24 & العلاوي \\
\hline 21.6 & 8 & 37 & مدينة الصدر \\
\hline 6.52 & 3 & 46 & الدورة \\
\hline 15.1 & 21 & 139 & الحسينية \\
\hline 3.12 & 1 & 32 & الغزالية \\
\hline 26.98 & 17 & 63 & الراشدية \\
\hline 15.8 & 79 & 500 & المجموع \\
\hline
\end{tabular}

جدول(6) أعداد ونسب الإصابة بطفيلي Cryptosporidium وحسب العمرحيث سجلت اعلى نسبة اصابة في الفئة العمرية اقل من

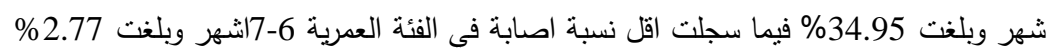


المجلة الطبية البيطرية العراقية, المجلد 33 ,العدد 2 , السنة 2009

\begin{tabular}{|c|c|c|c|}
\hline النسبة المئوية\%\% & عدد الحيو انات المصـابة & عدد الحيو انات & العمر بالثهر \\
\hline 34.95 & 43 & 123 & اقل من شهر \\
\hline 17.64 & 9 & 51 & $2<-1$ \\
\hline 12.72 & 7 & 55 & $3<-2$ \\
\hline 14.08 & 10 & 71 & $4<-3$ \\
\hline 7.5 & 3 & 40 & $5<-4$ \\
\hline 8.82 & 3 & 34 & $6<-5$ \\
\hline 2.77 & 1 & 36 & $7<-6$ \\
\hline 4.44 & 2 & 45 & $8<-7$ \\
\hline 3.57 & 1 & 28 & سنة \\
\hline$\% 0$ & 0 & 17 & > > سنتين \\
\hline 15.8 & 79 & 500 & المجموع \\
\hline
\end{tabular}

جدول (7) أعداد ونسب الإصابة بطفيلي Cryptosporidium وحسب الجنس ولم تسجل فروق معنوية بين الجنسين

\begin{tabular}{|c|c|c|c|}
\hline النسبة المئوية\% & عدد الحيوانات المصابة & عدد الحيوانات المفحوصة & الجنس \\
\hline 12.9 & 16 & 124 & ذكور \\
\hline 16.75 & 63 & 376 & إناث \\
\hline 15.8 & 79 & 500 & المجموع \\
\hline
\end{tabular}

جدول (8) أعداد ونسب الإصابة بطفيلي Cryptosporidium وحسب نوع البرازحيث لم تسجل فروق معنوية بين الجنسين

\begin{tabular}{|c|c|c|c|}
\hline النسبة المئوية\%\% & عدد الحيوانات المصابة & عدد الحيوانات المفحوصة & نوع البراز \\
\hline 18.61 & 43 & 231 & إسهال \\
\hline 11.9 & 15 & 126 & عجيني \\
\hline 14.68 & 21 & 143 & طبيعي \\
\hline 15.8 & 79 & 500 & المجموع \\
\hline
\end{tabular}

جدول (9) أعداد ونسب الإصـابة بطفيلي Cryptosporidium وحسب أنشهر الدراسـة حيث سجل شـهر نيسان اعلى نسبة اصـابة وبلغت 34.88\% فيما سجل شهر تموز اقل نسبة اصابة وبلغت 3.12 ونئ 


\begin{tabular}{|c|c|c|c|}
\hline النسبة المئوية\%\% & عدد الحيوانات المصابة & عدد الحيوانات المفحوصة & الثهر \\
\hline 7.14 & 4 & 56 & كانون الأول \\
\hline 11.66 & 7 & 60 & كانون الثاني \\
\hline 12.5 & 3 & 24 & شباط \\
\hline 21.6 & 8 & 37 & آذار \\
\hline 34.88 & 15 & 43 & 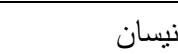 \\
\hline 26.98 & 17 & 63 & أيار \\
\hline 15.1 & 21 & 139 & حزيران \\
\hline 3.12 & 1 & 32 & تموز \\
\hline 6.52 & 3 & 46 & آب \\
\hline 15.8 & 79 & 500 & المجموع \\
\hline
\end{tabular}

المناقثة

أظهرت نتائج هذه الدراسـة إن نسبة الإصـابة الكلية بطفيلي البوغ الخبيء Cryptosporidium في الأغنام بلغت 15.8\% وجاءت

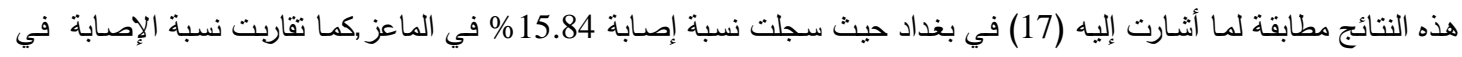

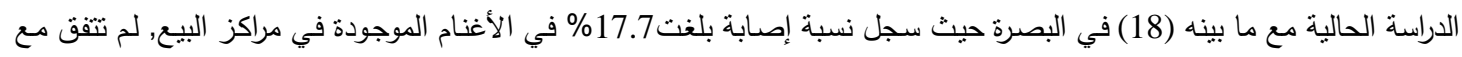

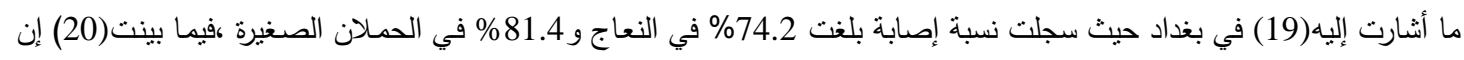
27.5 من الأغنام مصابة بطفيلي الابواغ الخبيئة في محافظة الديوانية.

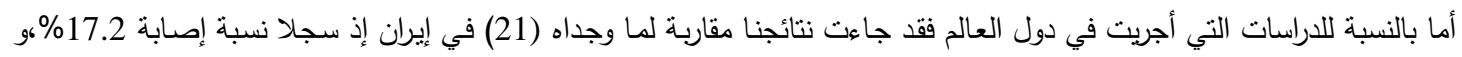

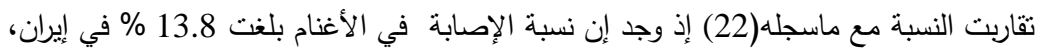

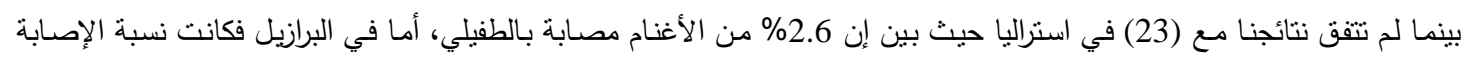
(24) $\% 3.7$

إن سبب الاختلافات في نسب الإصابة بالطفيلي في المناطق المختلفة من العالم وفي العراق قد يعود إلى الاختلاف في عدد الحيوانات المفحوصة وحجم العينة وطرائق الثربية والإدارة والظروف البيئية والمناخية التي تؤثر في مسنويات الاصـابة فضلا عن اختلاف الطرائق

النتخيصية المستخدمة في المختبر • بينت نتائج دراستتا إن هنالك اختلافات في نسب الإصابة حسب المواقع الجغرافية المشمولة بالدراسة إذ سجلت منطقة شمال بغداد أعلى نسب الإصابة لاسيما منطقة الشعب وبنسبة إصابة بلغت 34.88\%، فيما سجلت منطقة غرب بغداد اقل نسبة إصـابة وكانت أدناها في منطقة الغزالية بنسبة 3.12\% وجاءت هذه النتائج مطابقة لما أثنار إليه كل من(26,25) حيث ذكروا إن هنالك فروقات في نسبة

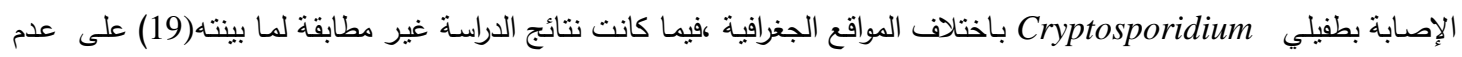
وجود فروقات معنوية في نسب الإصابة بين مناطق الدراسة المختلفة. قد يعود سبب الاختلاف في نسبة الإصابة باختلاف المواقع المشمولة بالدراسة إلى اختلاف ظروف فئل التربية حيث تكثر قطعان الأغنام

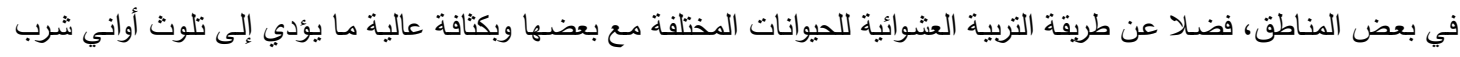
الماء والحليب والأعلاف بأكياس البيض فضلا عن عدم توافر شروط النظافة في أماكن التربية ما يؤدي إلى تراكم فضلات الحيوانات

التي تعد وسطا مناسبا لبقاء أكياس البيض حية ,فضلا عن تجمع الحشرات باعداد كبيرة والتي تعد ناقلا ميكانيكبا للطفيلي(27).

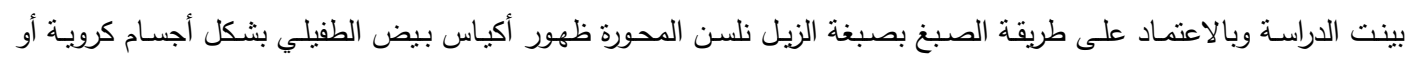

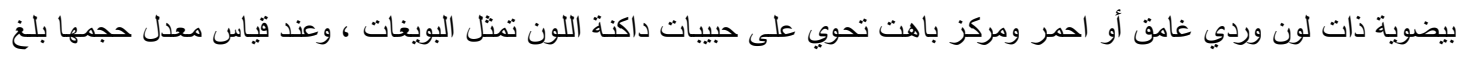




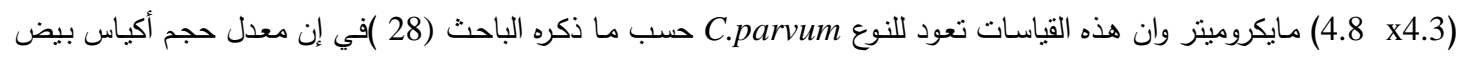
طفيلي C.parvum يبلغ (5x4.5) مايكروميتر . انققت هذه النتائج مـع ما سجلته (19) حيث وجدت إن معدل حجم أكياس بيض الطفيلي المعزولـة من الأغنام هو (4.8x4.2) مايكرومتر . مان.

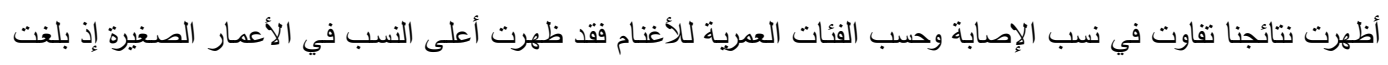

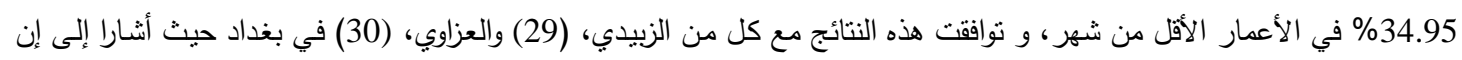

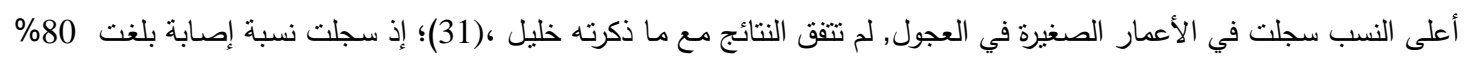

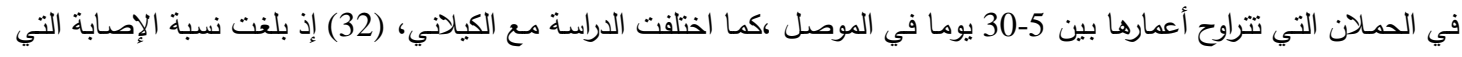

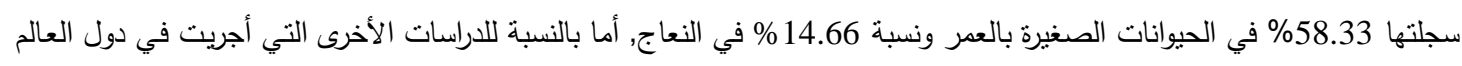

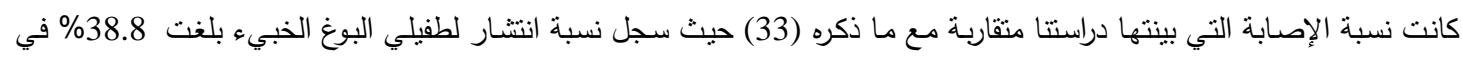

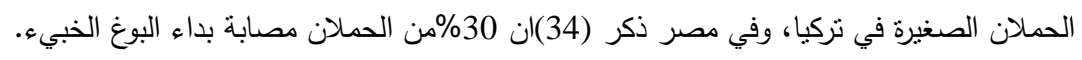

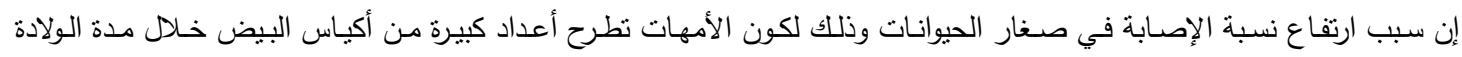

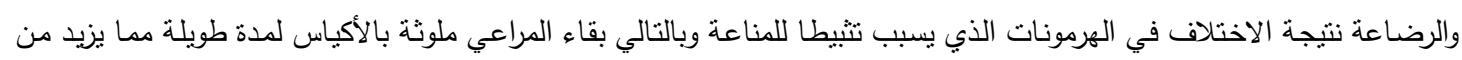

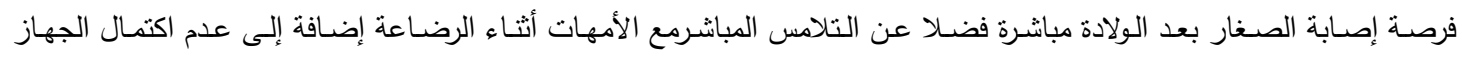

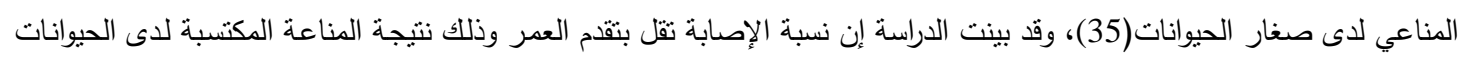
الكبيرة بتكرار الإصابة. لم تسجل دراستتا فرقا معنويا في نسب الإصابة بين الذكور والإناث وقد اتفقت هذه النتائج مع العديد من الدراسات في العراق التي

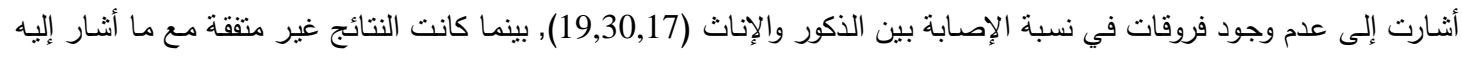
(29) في بغداد إذ سجل نسبة إصابة 39.2\% في الذكور ونسبة 28.6\% في الإنات فيمان فيما أوضحت ألكعبي، (2005) إن الإناث أكثر

إصابة من الذكور في محافظة الديوانية.

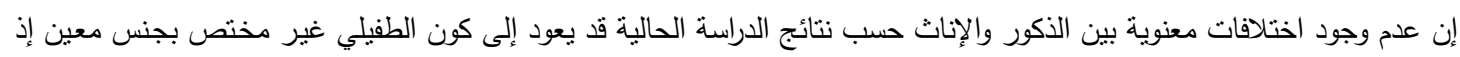

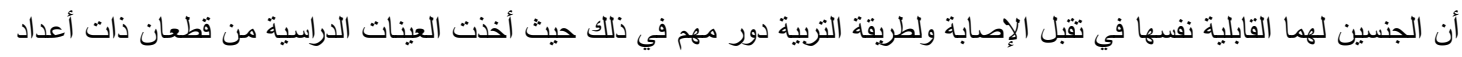

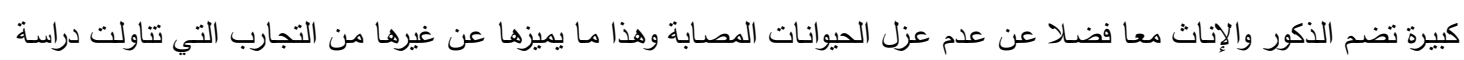
حيوانات مرباة في محطات نموذجية.

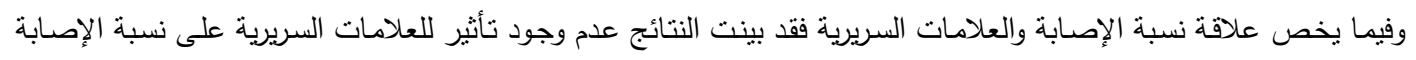

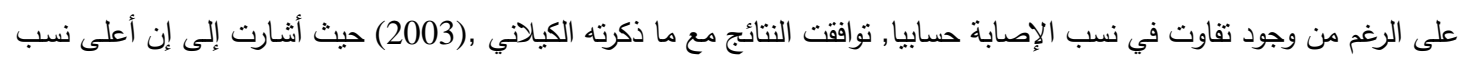

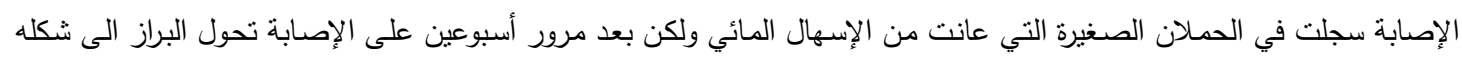

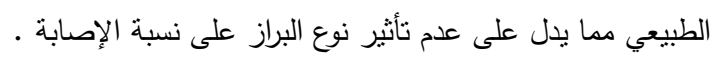

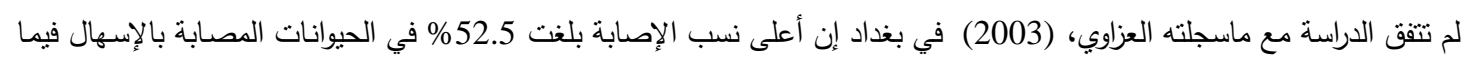

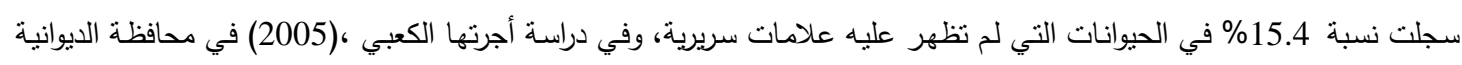

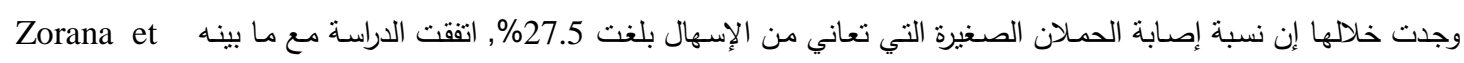

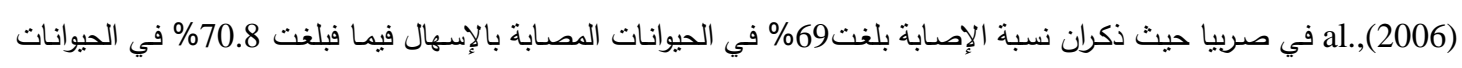
التي كان برازها طبيعيا. ان السبب في عدم وجود ثأثثر لنوع البراز على نسب الإصابة قد يعود إلى ضراوة العترة وعمر الحيوان والمقاومة التي يبديها كل حيوان

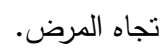

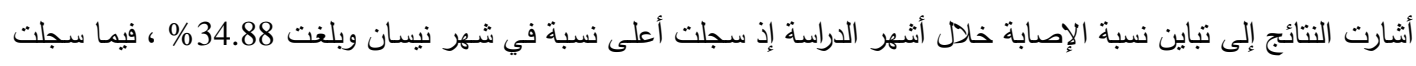

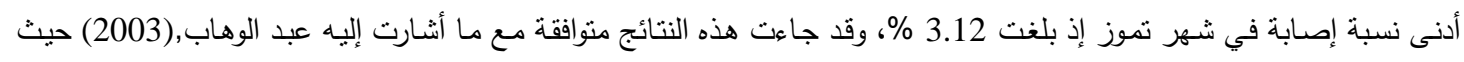

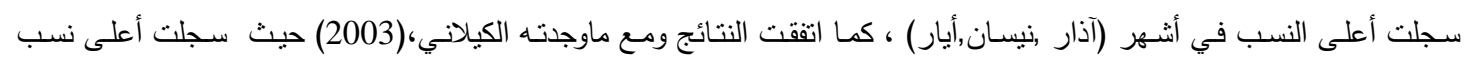

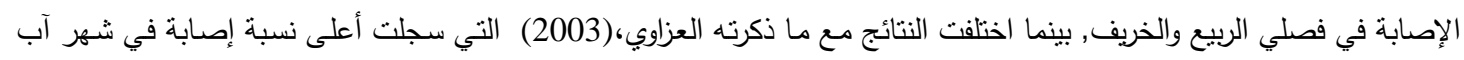

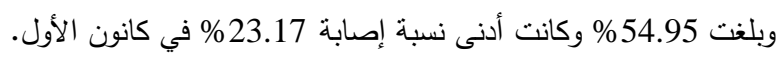

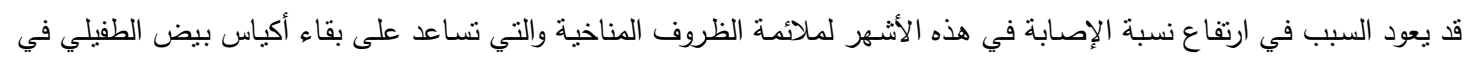

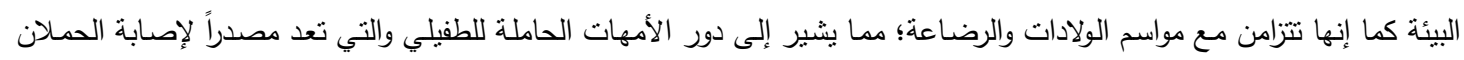


الصغيرة، ويعود الاختلاف الحاصل بين نتائج دراستتا والدراسات الأخرى إلى الاختلاف في عدد ونوع الحيوانات المفحوصة واختلاف الاشهر المشمولة بالدراسة حيث لم تتمل كافة أشهر السنة.

\section{References}

1. Chen,X.M.;Keithly,J.S.;Paya,C.V.and Larusso,N.F(2002) Cryptosporidiosis .NEJM.346(22):17231731.

2. Riggs ,M.W.; Mcneil ,M.R.; Perryman ,L.E.; Stone ,A.L.; Scherman ,M.S. and O’Connor ,R.M.(1999) Cryptosporidium parvum sporozoite pellicle antigen recognazid by anutralizing monoclonal antibody is a beta-mannosylated glycolipid.Infec.Immun.67(3):131- 1322.

3. Mosier,D.A.;Oberst,R.D.(2000) Cryptosporidiosis: aglobal challenge .Ann . N.Y. Acad.Sci.916:102-111.

4. Tzipor, S. and Griffiths, J.K.(2002) Natural history and biology of Cryptosporidium parvum.Parasitol.40:5-36.

5. Guerrant,R.L.(1999) Cryptosporidiosis: an emerging highly infectious threat. Emerg.Info.Dis.3:19.

6. Noordeen,F.;Horadagoda,N.U.;Faizal,A.C.;Rajapakes,R.P.;Razak,M.A.and

Arulkantham ,A.(2002) Infectivity of Cryptosporidium parvum isolated from asymptomatic adult goat to mice and goat kids.Vet.Parasitol .103 (3) :217-225

7. Causape,A.C.;Quilez,J.;Sanchez-Acedo,C.;Delcacho,E.and Bernad, L. F.(2002) Prevalence and analysis of potential risk factors for Cryptosporidium parvum infection in lambs in Zaragoza (northeastern Spain).Vet.Parasitol.104(4):287-298.

8. O'Donoghue,P.J.(1995) Cryptosporidium and cryptosporidiosis in man and animals .Int.J.parasitol.25:139-195.

9. Dupont ,H.L.; Chappell ,C.L.; Sterling ,C.R.; Okhuysen ,P.C.; Rose ,J.B. and Jarubowski ,W.(1995) The infectivity of Cryptosporidium parvum in healthy volunteers .N.Engl.J.Med.332(13): 855-859.

10. Dworkin,M.S.;Goldman,D.P.;Wells,T.G.;Kobayashi,T.M and Herwaldt „B. (1996) Cryptosporidiosis in Washington state :An outbreak with well water .J.Infec .Dis. 74:270-271.

11. Fayer,R.;Speer,C.A.and Duby,J.P.(1997b) General biology of Cryptosporidium and cryptosporidiosis of man and animal.Boca Raton FI CRC Press p.1-242.

12. Castro-Hermida,J.A.;Freire-Santos,F.;Oteiza-Lpez,A.M.;Vergara Castiblanco and AresMazes,M.E.(2000) Invitro and in vivo efficacy of lasalocid for treatment of Cryptosporidiosis. Vet.Parasitol.90:265-270.

13. Rossignol,J.F.;Ayoub,A.and Ayers,M.S.(2001) Tretment of diarrhea caused by Cryptosporidium parvum: aprospective randomized,double-blid,placebo-controlled study of nitazoxanide.J.Infec.Dis.184(1):103-106.

14. Beaver ,P.C. and Jung ,R.C.(1985) Animal agents and vectors of human diseases .(5 $5^{\text {th }}$ ed.) Lea and Febiger .p 249.

15. Chermette,R.and Boufassa,Q.S.(1988) Cryptosporidiosis a cosmopolitan diseases in animals and man.Epizodies.Fran. $2^{\text {nd }}$.(ed). Office Internatinal.Des. Epizodies .France.

16. Levine ,N.D.(1961) Protozoan parasitio.of domestic animals and of man.Burgess publishing company,Minnesota,USA.PP:118-122

17. Rasheed ,R.N.(1997) Cryptosporidiosis in Iraqi goat kids. The Veterinarian . 1(6):1-5.

18. Ali,N.H. (1998) Prevalence of cryptosporidiosis with zoonotic aspect .M.Sc. Thesis, Collage of Medicine,University of Basrah.

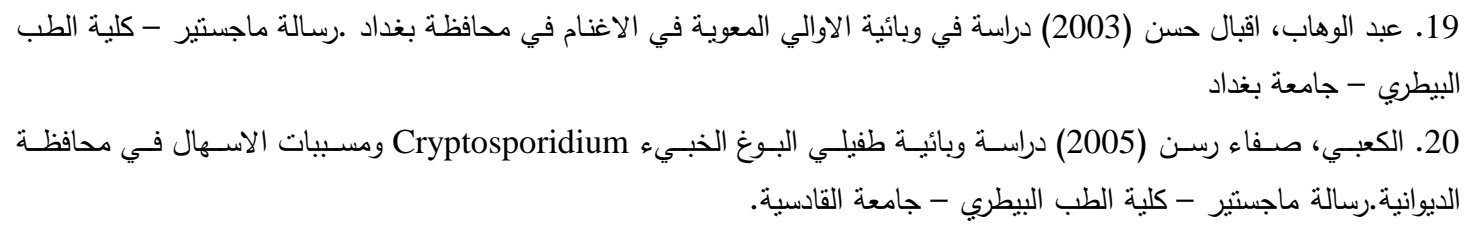

21. Nouri,M.and Karami,M.(1991) Asymptomatic cryptosporidiosis in nomadic shepherds and their sheep.J.Infec.23(3):331-333.

22. Harandi,F.M.and Ardakani,F.R.(2008) Cryptosporidium infection of sheep and goats in Kerman:Epidemiology and risk factor analysis.J.Vet .Res. 63 (1):47-51. 
23. Ryan,U.M.;Bath,C.;Robertson,I.;Read,C.;Elliot,A.;Mcinnes,L.;Trub,R.and Besier,B.(2005) Sheep may not be an important zoonotic reservoir for Cryptosporidium and Giardia parasites.Appl.Enviro.Microbiol.71(9):4992-4997.

24. Tembue,A.A.M.;Alves,L.C.;Borges.J.C.G.;Faustino,M.A.Da Gloria.and Machado,E.L.C.(2006) Cryptosporidium spp.in sheep in ibimirim district, pernambuco state,Brazil.Cienc.Vet.Trop.Recife-PE.9(10):41-43.

25. Ruest,N.;Faubert,G.M.and Coutuer,Y.(1998) Prevalence and giografical distribution of Giardia spp.and Cryptosporidium spp.in dairy farms in Quebec.Can.Vet.j.39(11):697-700.

26. Causape,A.C.;Quilez,J.;Sanchez-Acedo,C.;Delcacho,E.and Bernad , L. F.(2002) Prevalence and analysis of potential risk factors for Cryptosporidium parvum infection in lambs in Zaragoza (northeastern Spain).Vet.Parasitol.104(4):287-298.

27. Mohammed ,H.O.;Wade,S.E.and Schaaf,S.(1999) Risk factors associated with Cryptosporidium parvum infection in dairy cattle in south astern New York state.Vet.Parasitol.83(1):1-13.

28. Hart,G.(1999) Cryptosporidiosis.In:Protozoal disease. Gilles , H.M.(ed). $1^{\text {st }}$.Published. Amember of the holder headline group.London.pp592-602.

29. الزبيدي، محمد ثابت (1994) دراسة داء الابواغ الخبيئة في العجول .رسالة ماجستير - كلبة الطب البيطري - جامعة بغداد.

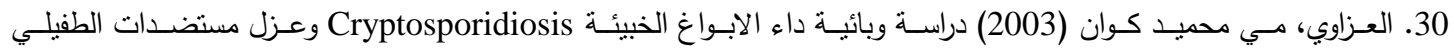

وتتخيصها واستعمال بعض مستخلصات النبات الطبية كمحاولة للعلاج. اطروحة دكتوراه - كلية الطب البيطري - جامعة بغداد.

31. خليل، ليان ياسين (2000) مقارنـة كفاءة بعض الاختبارات التشخيصية لداء الابواغ الخبيئة في الحملان والاطفال في محافظة

نينوى.رسالة ماجستير -كلية الطب البيطري - جامعة الموصل.

32. الكيلاني، بـان عبد الوهاب (2003) دراسـة وبائية وتشخيصية لطفيلي الابواغ الخبيئة في الانسـان والحيوان في قريـة الذهب

الابيض ـ اطروحة دكتوراه - كلية الطب البيطري - جامعة بغداد بلاد

33. Sari ,B.; Arslan ,M.O.; Gicik ,Y.; Kara ,M. and Tasci ,G.T.(2008) The prevaleance of Cryptosporidium species in diarrhoeic lambs in Kars province and potential risk factors.Trop.Anim.Health.Prod.

34. El.Wahed,M.A.(1999) Cryptosporidium infection among sheep in Qalubia governorate.Egypt.J.Egy.Soc.Parasitol.29:113-118.

35. Anderson,B.C. (1991) Experimental infection in mice of Cryptosporidium muris isolated from a camel. J. Protozol.38:165-175.

36. Zorana,M.;Katic-Radivojevic,S.and Kulisic,Z.(2006) Cryptosporidium infection in lambs and goat kids in Serbia.Vet.Acta.56(1):49-54. 